\title{
APLICAÇÃO DE MÉTODOS NUMÉRICOS PARA ESTIMATIVA DE VARIÁVEIS PSICROMÉTRICAS
}

\author{
JARBAS H. DE MIRANDA ${ }^{1}$, LUIZ R. ANGELOCCI ${ }^{2}$, KÉSIA O. DA SILVA ${ }^{3}$, \\ SERGIO N. DUARTE ${ }^{4}$, NILSON A. VILLA NOVA ${ }^{5}$
}

\begin{abstract}
RESUMO: O ar atmosférico é formado por uma composição de ar seco e vapor d'água, cujos valores da quantidade de vapor, em termos quantitativos, são variáveis em função dos elementos climáticos, tais como: temperatura e pressão. Portanto, para estimar os valores de umidade relativa do ar, são necessárias informações completas de séries de dados de temperatura do ar $\left(\mathrm{T}_{\mathrm{ar}}\right)$, pressão atual de vapor $\left(\mathrm{e}_{\mathrm{a}}\right)$ e temperatura de bulbo úmido $\left(\mathrm{T}_{\mathrm{u}}\right)$. $\mathrm{O}$ trabalho apresenta como objetivo a elaboração de rotina computacional para encontrar valores da temperatura do termômetro de bulbo úmido $\left(\mathrm{T}_{\mathrm{u}}\right)$ em função de outras variáveis, para uma série de dados obtidos, mediante a aplicação de métodos numéricos (Método de Newton-Raphson e Método da Secante), para fins de complementação de uma série de dados diários para Piracicaba - SP. Verificou-se que os métodos numéricos de Newton-Raphson e da Secante apresentaram bom desempenho na simulação dos resultados, porém o método da Secante apresentou-se como o melhor para encontrar os valores de $\mathrm{T}_{\mathrm{u}}$, mediante a resolução numérica da equação psicrométrica.
\end{abstract}

PALAVRAS-CHAVE: métodos numéricos, temperatura de bulbo úmido, umidade relativa.

\section{APPLICATION OF NUMERIC METHODS FOR PSYCHROMETRIC VARIABLES ESTIMATION}

\begin{abstract}
The atmospheric air is formed by a composition of dry air and water vapour, whose values of the vapour amount, in quantitative terms, are variable in function of climatic elements such as temperature and pressure. Therefore, to estimate the air relative humidity is necessary complete information's of historical data series of air temperature $\left(\mathrm{T}_{\mathrm{ar}}\right)$, vapour pressure $\left(\mathrm{e}_{\mathrm{a}}\right)$ and wet-bulb temperature $\left(T_{u}\right)$. Therefore, these values were used to calculate $T_{u}$ in order to complete the daily data series for Piracicaba - SP, Brazil. This work had the objective of elaborating a computational routine to estimate values of the wet-bulb temperature $\left(\mathrm{T}_{\mathrm{u}}\right)$ as a function of climate variables, to obtain data series, based on numeric methods (Newton-Raphson Method and Secant Method). The results showed that the numeric methods of Newton-Raphson and Secant presented good estimates of $\mathrm{T}_{\mathrm{u}}$, however the Secant method showed as the best numeric method to be applied for the numeric solution of the psychrometric variables.
\end{abstract}

KEYWORDS: numeric methods, wet-bulb temperature, relative humidity.

\section{INTRODUÇÃO}

O desenvolvimento de técnicas de manejo de irrigação requer o conhecimento de quando, quanto e como aplicar água, sendo dependentes das condições atmosféricas locais; o conhecimento dessas variações tem grandes aplicações no meio agrícola, principalmente em se tratando das necessidades hídricas das culturas (CASTELLVÍ et al., 1996). O ar atmosférico é composto por uma mistura de ar seco e vapor d'água e, em certas condições de temperatura e pressão, essa composição é capaz de conter quantidades variáveis de vapor d'água, pois normalmente o ar

\footnotetext{
${ }^{1}$ Eng $^{\mathrm{o}}$ Agrônomo, Prof. Dr., Departamento de Ciências Exatas, Escola Superior de Agricultura "Luiz de Queiroz" - ESALQ/USP, Av. Pádua Dias, 11 Caixa Postal 9, Piracicaba - SP, Fone: (0XX19) 3429.4283, ramal: 210, jhmirand@esalq.usp.br

${ }^{2}$ Prof. Associado, Departamento de Ciências Exatas, ESALQ/USP, Piracicaba - SP, 1rangelo@esalq.usp.br

${ }^{3}$ Profa. Dra., Departamento de Engenharia Rural, ESALQ/USP, Piracicaba - SP, kosilva@uol.com.br

${ }^{4}$ Prof. Dr., Departamento de Engenharia Rural, ESALQ/USP, Piracicaba - SP, snduarte@esalq.usp.br

${ }^{5}$ Prof. Associado, Departamento de Ciências Exatas, ESALQ/USP, Piracicaba - SP, navnova@esalq.usp.br

Recebido pelo Conselho Editorial em: 30-1-2006
}

Aprovado pelo Conselho Editorial em: 30-8-2006

Eng. Agríc., Jaboticabal, v.26, n.3, p.686-694, set./dez. 2006 
encontra-se não-saturado e, portanto, capaz de reter maior quantidade de vapor d'água (SILVA, 2002). Para que ocorra essa retenção, é necessário que a água passe da fase líquida para a fase de vapor, cuja mudança demanda certa quantidade de energia, a qual é retirada do ar, resfriando-o (SILVA, 2002). Nesse caso, pode-se definir a evaporação como sendo a transição entre fase líquido-vapor (vaporização), ou seja, a uma temperatura menor que a de ebulição e possuindo as moléculas da interface atuando no processo, caracterizando-se, portanto, como um fenômeno de superfície.

Dados meteorológicos do ambiente freqüentemente podem ser apresentados por diferentes maneiras, em termos de variações da quantidade de vapor d'água na atmosfera, na escala vertical e temporal, e normalmente são obtidos por diferentes instrumentos (JUPP, 2003).

O conhecimento de propriedades psicrométricas é fundamental para projetar sistemas de controle ambiental para plantas, animais e seres humanos (SINGH et al., 2002).

Normalmente, as pesquisas sobre psicrometria são feitas utilizando-se de um instrumento denominado psicrômetro, o qual se trata, basicamente, de um conjunto de termômetros de bulbo seco e úmido (coberto por um tecido imerso em água) (DIAS, 2001). Quando o termômetro de bulbo úmido é colocado em uma corrente de ar, a água se evapora do tecido, até que atinja uma temperatura de equilíbrio, chamada temperatura do termômetro de bulbo úmido $\left(\mathrm{T}_{\mathrm{u}}\right)$. Esse processo não é de saturação adiabática, a qual define a $T_{u}$ em termos dinâmicos, mas trata-se de transferência de calor e massa simultâneos no bulbo úmido (BEYER, 1998, citado por DIAS, 2001).

Portanto, por intermédio do psicrômetro, podem ser obtidas as temperaturas de bulbo úmido e da temperatura do ar (bulbo seco), tendo sido o método mais comum para obter informações sobre o vapor d'água em estações meteorológicas tradicionais. Com essas duas propriedades psicrométricas, juntamente com a pressão atmosférica, é possível calcular várias propriedades psicrométricas, tais como: a pressão exercida pelo vapor sobre a massa líquida, chamada de pressão atual de vapor $\left(\mathrm{e}_{\mathrm{a}}\right)$ (COSTA, 2003), e a umidade relativa do ar, que é a razão entre a pressão atual de vapor e a pressão de saturação de vapor (es) (MONTEITH \& UNSWORTH, 1990).

Porém, a determinação de $\mathrm{T}_{\mathrm{u}}$, que é uma medida da quantidade de umidade do vapor d'água contido no ar, torna-se algumas vezes difícil e, diante das equações disponíveis, pode ser obtida mediante tentativas ou iterações, realizadas por métodos numéricos, tais como: Newton-Raphson e Secante. Pode-se dizer, em termos gerais, que são métodos bastante difundidos e com ampla aplicação em diferentes áreas da Engenharia Agrícola. O método da secante é uma variação do método de Newton-Raphson, o qual converge quadraticamente para raízes simples, enquanto o método da secante converge linearmente para a mesma situação (DÍEZ, 2003).

Portanto, o objetivo do presente trabalho foi desenvolver rotina computacional, em Visual Basic 6.0, aplicando métodos numéricos (Método de Newton-Raphson e Método da Secante) para encontrar valores da temperatura de bulbo úmido em função de outros parâmetros (pressão parcial de vapor d'água, constante psicrométrica, pressão do local e temperatura do ar), para completar uma série de dados meteorológicos obtidos para Piracicaba - SP.

\section{MATERIAL E MÉTODOS}

Os dados meteorológicos de temperatura do ar $\left(\mathrm{T}_{\mathrm{ar}}\right)$ e pressão atual de vapor $\left(\mathrm{e}_{\mathrm{a}}\right)$, utilizados neste trabalho, foram obtidos por uma sonda de temperatura e umidade relativa do ar, do tipo HMP35C (Campbell Scientific, Inc.), instalada no posto agrometeorológico da Escola Superior de Agricultura "Luiz de Queiroz" - ESALQ/USP, localizado a $22^{\circ} 42^{\prime} 30^{\prime}$ ' S e $47^{\circ} 38^{\prime} 00^{\prime}$ " W, a 546 m de altitude, em Piracicaba - SP.

O equipamento coletou dados diários de temperatura do ar $\left(\mathrm{T}_{\mathrm{ar}}\right)$ e da pressão atual de vapor $\left(\mathrm{e}_{\mathrm{a}}\right)$ em intervalos de 15 minutos, iniciando-se à $0 \mathrm{~h}$ do dia 1 - $-1-2003$ até às $7 \mathrm{~h} 30$ do dia 8-12-2003, totalizando-se 32.767 valores, utilizados para a simulação. 


\section{Pressão de saturação de vapor}

Para calcular os valores de pressão de saturação de vapor em situações práticas, utiliza-se da aproximação e derivações de TETENS (1930), amplamente utilizadas em Agrometeorologia, expressa por MURRAY (1967), dada por:

$$
\mathrm{e}_{\mathrm{s}}=0,610810^{\frac{7,5 \mathrm{Ts}}{237,3+\mathrm{Ts}}}
$$

em que,

$\mathrm{e}_{\mathrm{s}}$ - pressão de saturação de vapor d'água, $\mathrm{kPa}, \mathrm{e}$

$\mathrm{T}_{\mathrm{s}}$ - temperatura do psicrômetro de bulbo seco, ${ }^{\circ} \mathrm{C}$.

\section{Pressão parcial de vapor d’água}

A pressão exercida pela massa atual de vapor d'água existente na atmosfera é definida pelo símbolo $\mathrm{e}_{\mathrm{a}}$. A pressão parcial de vapor $\left(\mathrm{e}_{\mathrm{a}}\right)$ varia desde zero, para o ar totalmente seco, até ao valor máximo denominado de pressão de saturação de vapor d’água $\left(e_{s}\right)$ (PEREIRA et al., 2001), dada por:

$$
\mathrm{e}_{\mathrm{a}}=\mathrm{e}_{\mathrm{su}}-\operatorname{cpP}\left(\mathrm{T}_{\mathrm{s}}-\mathrm{T}_{\mathrm{u}}\right)
$$

em que,

$\mathrm{e}_{\mathrm{a}-}$ - pressão atual de vapor d'água, $\mathrm{kPa}$;

$\mathrm{e}_{\mathrm{su}}$ - pressão de saturação de vapor d'água, com base na temperatura do termômetro de bulbo úmido, ${ }^{\circ} \mathrm{C}$;

cp - constante psicrométrica $\left(0,0008{ }^{\circ} \mathrm{C}^{-1}\right.$, psicrômetro não-aspirado);

$\mathrm{P}$ - pressão atmosférica local, $\mathrm{kPa}$;

Ts - temperatura do termômetro de bulbo seco, ${ }^{\circ} \mathrm{C}$, e

$\mathrm{Tu}$ - temperatura do termômetro de bulbo úmido, ${ }^{\circ} \mathrm{C}$.

Substituindo-se a expressão de pressão de saturação de vapor d’água, com base na temperatura do termômetro de bulbo úmido, na eq.(2), obtém-se a eq.(3), dada por:

$$
\mathrm{e}_{\mathrm{a}}=0,610810^{\frac{7,5 \mathrm{Tu}}{237,3+\mathrm{Tu}}}-\operatorname{cp~P}\left(\mathrm{T}_{\mathrm{s}}-\mathrm{T}_{\mathrm{u}}\right)
$$

A partir da eq.(3), chega-se ao problema a ser resolvido, uma vez que a série de dados, coletada na estação, disponibiliza apenas a pressão atual de vapor $(\mathrm{kPa})$, a temperatura do termômetro de bulbo seco $\left({ }^{\circ} \mathrm{C}\right)$, a constante psicrométrica $(\mathrm{cp})$ e a pressão do local $(\mathrm{P})$, restando somente a temperatura do termômetro de bulbo úmido $\left(\mathrm{T}_{\mathrm{u}}\right)$, utilizando-se de métodos numéricos para encontrar os valores de $\mathrm{T}_{\mathrm{u}}$ e completar a série de dados e ser disponibilizada para os usuários, mediante a resolução numérica da eq.(4), por meio de processos iterativos $\left(f\left(T_{u}\right)=e_{a}\right)$.

$$
f\left(T_{u}\right)=\left[0,610810^{\frac{7,5 T_{u}}{237,3+T_{u}}}-\operatorname{cp~P}\left(T_{s}-T_{u}\right)\right]
$$

\section{Métodos numéricos}

Foram aplicados dois métodos numéricos: o método de Newton-Raphson e o método da Secante. O método de Newton-Raphson é muito útil para determinar raízes de funções. A seqüência gerada dessa maneira converge para uma raiz de $f\left(T_{u}\right)$, em que $T u_{1}$ é um valor inicialmente atribuído à raiz. Geometricamente, o método de Newton-Raphson funciona traçando tangentes à curva nas aproximações de $\mathrm{T}_{\mathrm{u}}$ e tomando a próxima aproximação dada pela interseção da reta tangente com o eixo de $\mathrm{T}_{\mathrm{u}}$ (Figura 1 ). 


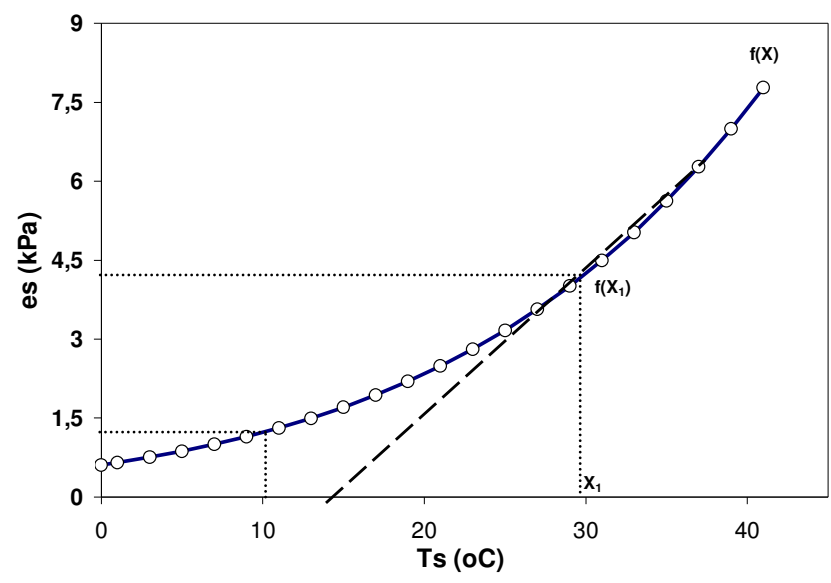

(a)

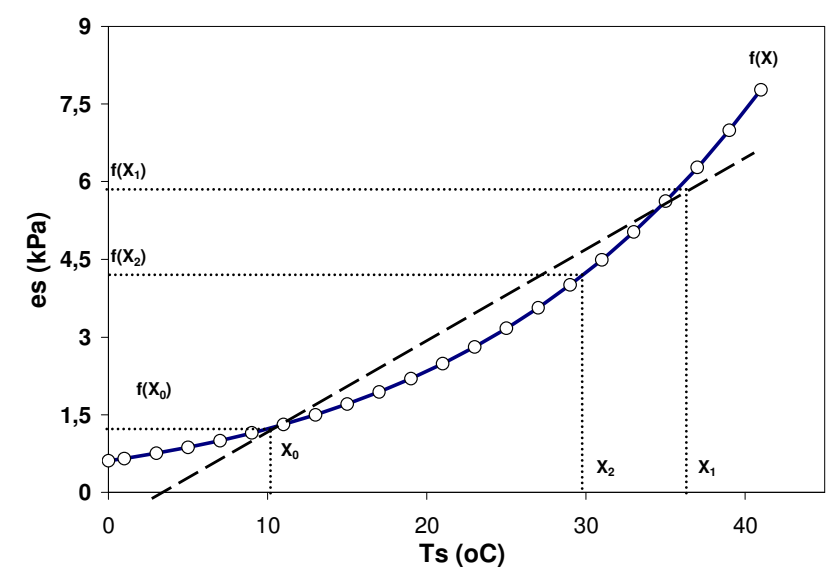

(b)

FIGURA 1. Representação esquemática do método de Newton-Raphson (a) e do método da Secante (b).

O método de Newton-Raphson é baseado num processo de iteração dado por:

$$
\mathrm{Tu}_{\mathrm{i}+1}=\mathrm{Tu} \mathrm{u}_{\mathrm{i}}-\frac{\mathrm{f}(\mathrm{Tu})}{\mathrm{f}^{\prime}(\mathrm{Tu})}
$$

Cada método apresenta uma característica própria para a resolução e, dentre essas, pode-se ressaltar que existe uma desvantagem do Método de Newton-Raphson, que é a obtenção da derivada da função $\left(f^{\prime}\left(T_{u}\right)\right)$, cujas expressões são dadas pelas eqs.(5) e (6):

$$
\begin{aligned}
& f^{\prime}(T u)=\frac{d}{d T_{u}}\left[e_{a}-0,610810^{\frac{7,5 t u}{237,3+t u}}+\operatorname{cp~P}\left(T_{s}-T_{u}\right)\right] \\
& f^{\prime}(T u)=-0,6108100^{7,5 \frac{T_{u}}{237,3+T_{u}}}\left[\frac{7,5}{237,3+T_{u}}-7,5 \frac{T_{u}}{\left(237,3+T_{u}\right)^{2}}\right] \operatorname{Ln}(10)-c p P
\end{aligned}
$$

Diante disso, elaborou-se uma rotina computacional capaz de determinar os valores de $T_{u}$ para uma série extensa de dados, com rapidez e precisão, utilizando-se, para tal, dos métodos numéricos de Newton-Raphson e Secante. O fluxograma da rotina computacional para o método de Newton-Raphson é mostrado na Figura 2.

O outro método aplicado foi o da Secante, que contorna de certa forma a dificuldade do método de Newton-Raphson, substituindo-se a derivada da função por uma aproximação. Pode-se, portanto, descrever que, sendo dadas duas aproximações $\mathrm{Tu}_{\mathrm{i}}$ e $\mathrm{Tu}_{\mathrm{i}+1}$, traça-se uma reta secante que passa por $\left(T u_{i}, f\left(T u_{i}\right)\right)$ e $\left(T u_{i+1}, f\left(T u_{i+1}\right)\right)$. Nesse caso, obtém-se a interceção com o eixo x em $\left(\left(\varphi\left(\mathrm{Tu}_{\mathrm{i}+1}\right), 0\right)\right.$. Faz-se $\mathrm{Tu}_{\mathrm{i}}$ assumir o valor de $\mathrm{Tu}_{\mathrm{i}+1}$, e $\mathrm{Tu}_{\mathrm{i}+1}$ receber o valor de $\varphi\left(\mathrm{Tu}_{\mathrm{i}+1}\right)$. Repete-se esse processo até obter a precisão estabelecida, ou seja, o fato de que os dois métodos numéricos são executados até satisfazer uma condição de erro (estabelecido pelo usuário), que nada mais é do que o estabelecimento de uma diferença entre a leitura calculada $\left(\mathrm{Tu}_{\mathrm{i}+1}\right)$ e a obtida, anteriormente $\left(\mathrm{Tu}_{\mathrm{i}}\right)$. No caso das duas rotinas, o erro estabelecido foi de $10^{-8}$. Esse processo para a determinação de aproximações das raízes de uma equação é semelhante ao método de Newton-Raphson. Nele, a equação da tangente é substituída pela equação da secante que corta a curva da função em dois pontos cujas abscissas definem um intervalo no qual está contida a raiz $\left(\mathrm{T}_{\mathrm{LI}}=\right.$ temperatura limite inferior; $\mathrm{T}_{\mathrm{LS}}=$ temperatura limite superior). $\mathrm{O}$ fluxograma da rotina computacional para o método da Secante é mostrado na Figura 3. 


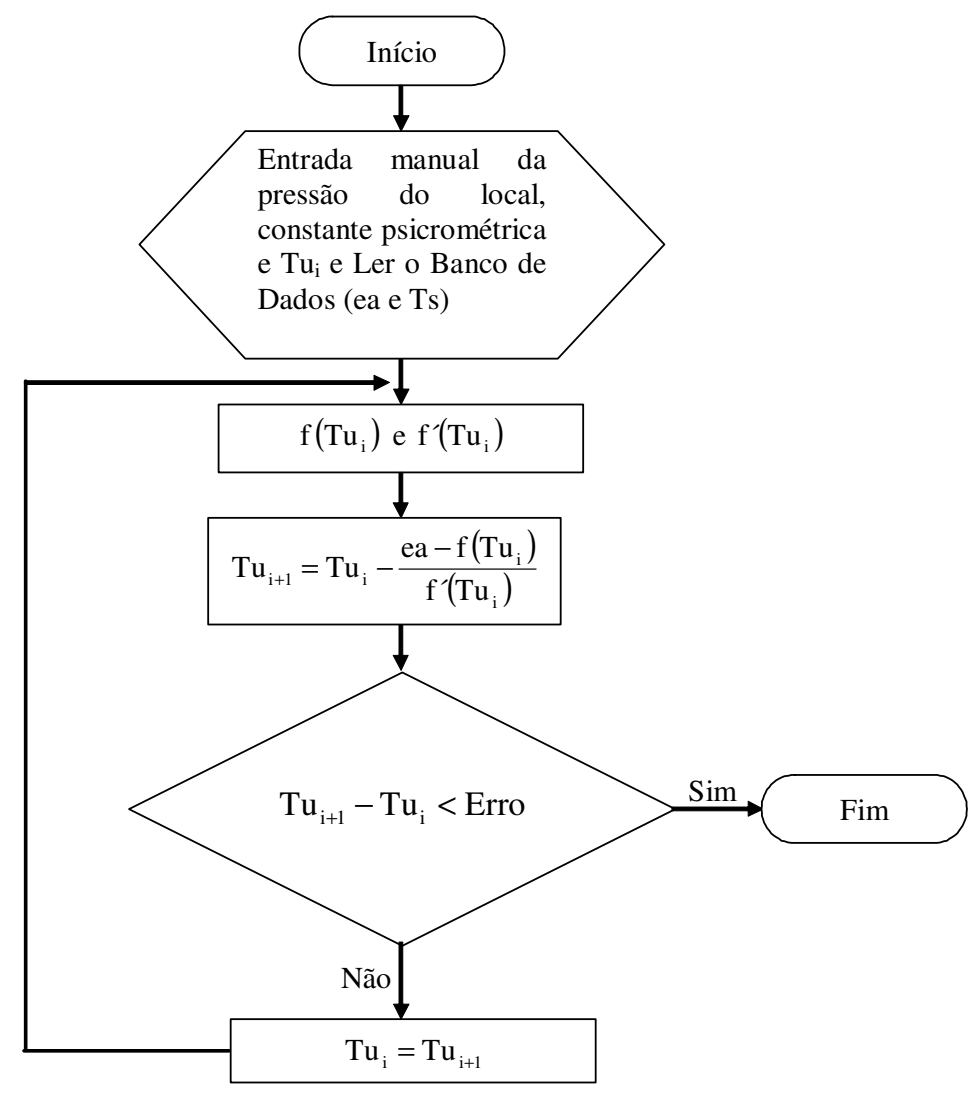

FIGURA 2. Fluxograma do método de Newton-Raphson.

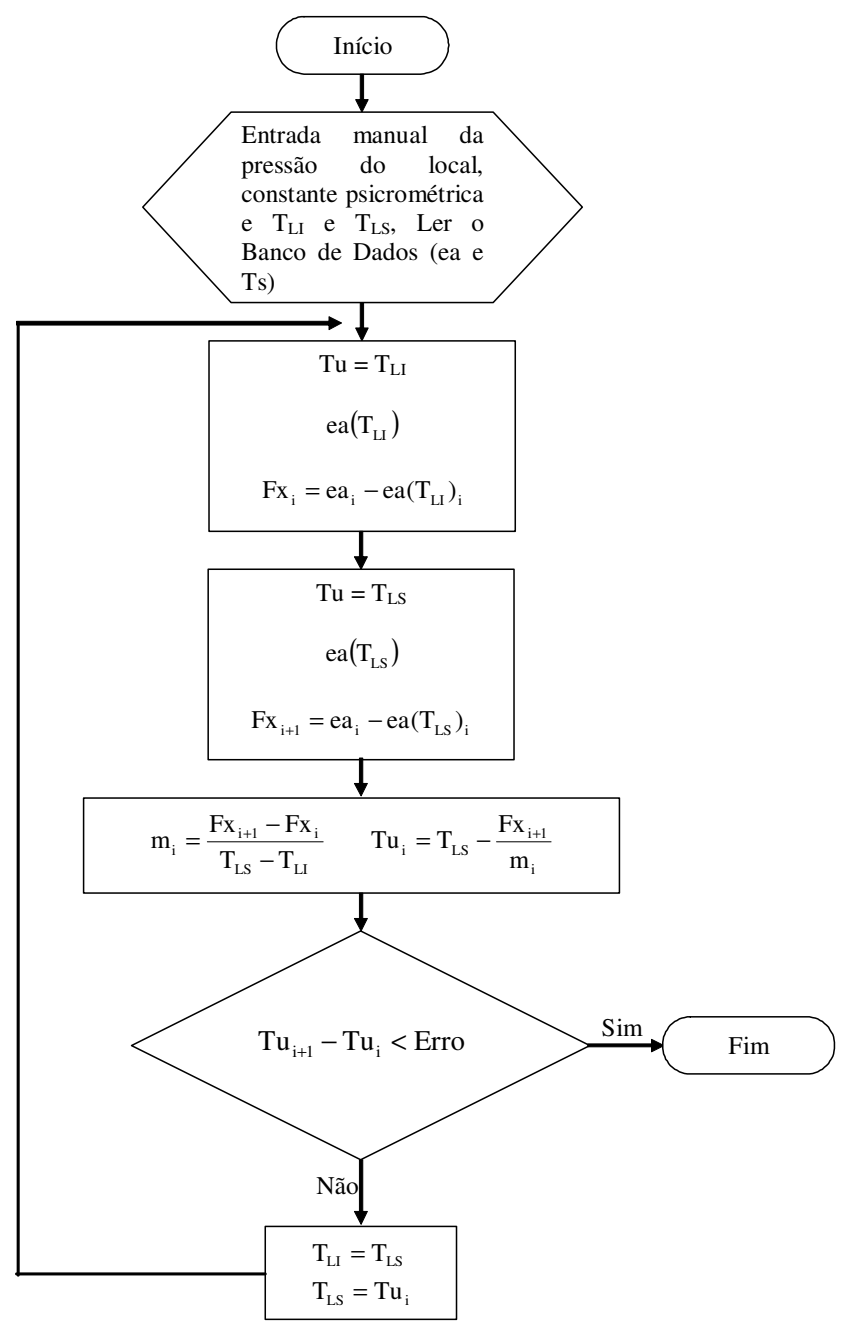

FIGURA 3. Fluxograma do método da Secante. 


\section{Rotina computacional}

Desenvolveu-se um programa em Visual Basic 6.0, que permite ao usuário a opção de aplicação de um dos métodos numéricos descritos anteriormente, para encontrar o valor de $\mathrm{T}_{\mathrm{u}}$ correspondente ao valor da pressão atual de vapor $(\mathrm{kPa})$, registrada pela estação automática, conforme Figura 4.

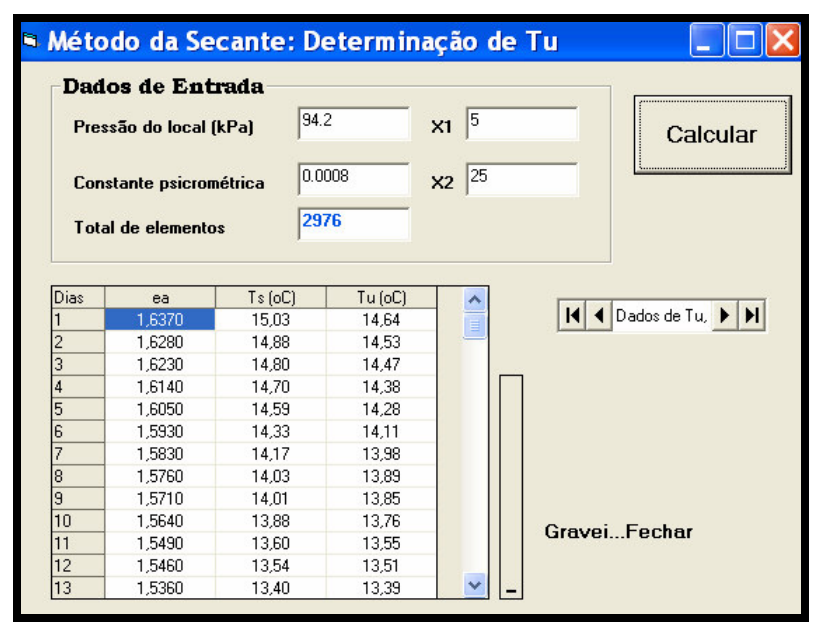

(a)

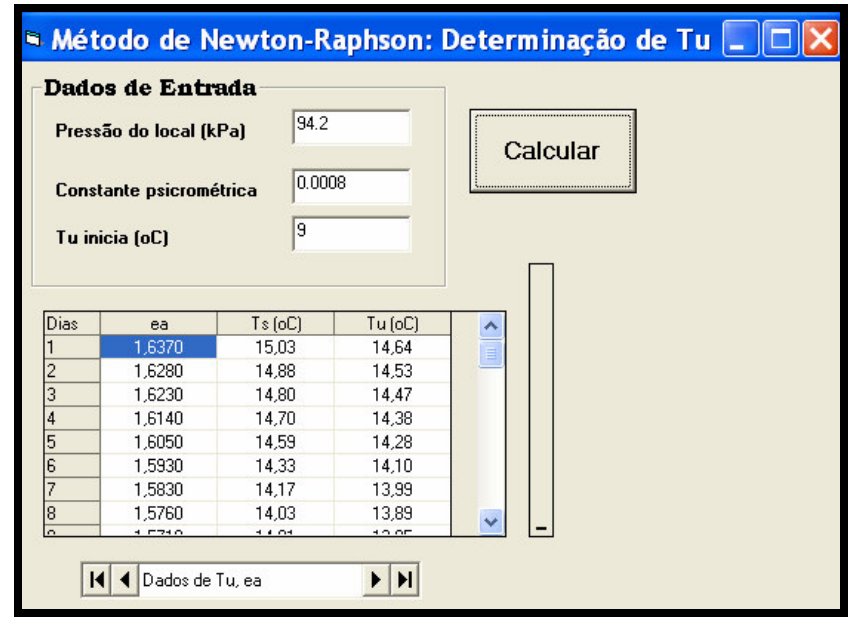

(b)

FIGURA 4. Tela da rotina computacional para a estimativa de $\mathrm{T}_{\mathrm{u}}$, utilizando-se do Método da Secante (a); tela da rotina computacional para a estimativa de $\mathrm{T}_{\mathrm{u}}$, utilizando-se do Método de Newton-Raphson (b)

\section{RESULTADOS E DISCUSSÃO}

Nota-se que os métodos de Newton-Raphson e Secante apresentaram bom desempenho na simulação dos resultados, porém, para algumas situações, por exemplo, 263 valores de Tu foram nulos, para a série de 32.767 valores avaliados. Isso indica que o método de Newton-Raphson não convergiu para uma solução da equação, o que não aconteceu para o método da Secante. Verificase que, na série de dados, 5.467 valores apresentaram valores de $\mathrm{T}_{\mathrm{u}}$, obtidos pelo método de Newton-Raphson, superiores aos obtidos pelo da Secante (Figura 5).

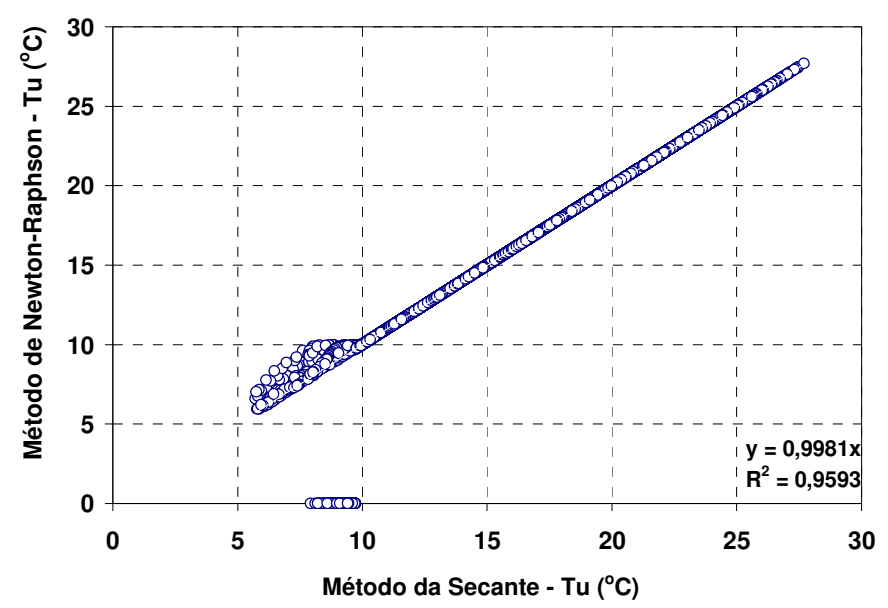

FIGURA 5. Correlação dos 32.767 valores de $\mathrm{T}_{\mathrm{u}}$ obtidos pelos métodos numéricos de NewtonRaphson e Secante.

O fato de o método de Newton-Raphson, em certas situações, não ter convergido para uma solução numérica, fez com que o número de iterações fosse maior do que o da Secante. Para a resolução dos valores de $\mathrm{T}_{\mathrm{u}}$, o método de Newton-Raphson totalizou média de 11,75 iterações para encontrar o resultado da equação, ao passo que o método da Secante apresentou média de 3,11 iterações. Porém, em termos de tempo de máquina, pelo método de Newton-Raphson, foram gastos 
um total de $28 \mathrm{~s}$, enquanto, pelo método da Secante, foram gastos aproximadamente $55 \mathrm{~s}$. Isso evidencia que o método de Newton-Raphson é mais eficiente que o da Secante para a resolução da equação, porém, devido a algumas situações, o referido método não encontrou as soluções da equação.

$\mathrm{Na}$ tentativa de melhor esclarecimento desse comportamento do Método de NewtonRaphson, elaborou-se um gráfico com os valores de temperatura do ar $\left(\mathrm{T}_{\mathrm{ar}}\right)$ e de pressão parcial de vapor $\left(\mathrm{e}_{\mathrm{a}}\right)$, para os referidos 263 valores, nos quais o Método de Newton-Raphson apresentou os valores nulos para $\mathrm{T}_{\mathrm{u}}$ (Tabela 1). Pôde-se observar que o comportamento dos valores de $\mathrm{T}_{\mathrm{ar}}$ e de $\mathrm{e}_{\mathrm{a}}$ para que o Método de Newton-Raphson não convergisse para uma solução, deve-se ao fato, principalmente, de que são valores baixos, especialmente entre o período de 7 de maio e 10 de setembro (Figura 6).

TABELA 1. Valores médios, mínimos e máximos da temperatura do ar (Tar) e pressão atual de vapor (ea) (263 dados), de uma série de 32.767 valores, do ano de 2003, para Piracicaba - SP.

\begin{tabular}{|c|c|c|}
\hline & \multicolumn{2}{|c|}{ Elementos Climáticos } \\
\hline & $\underset{{ }^{\circ} \mathrm{C}}{\text { Temperatura do } \operatorname{Ar}\left(\mathrm{T}_{\mathrm{ar}}\right)}$ & $\begin{array}{l}\text { Pressão Atual de Vapor }\left(\mathrm{e}_{\mathrm{a}}\right) \\
\mathrm{kPa}\end{array}$ \\
\hline Média & 11,03 & 1,0351 \\
\hline Valor Mínimo & 10,00 & 0,5450 \\
\hline Valor Máximo & 18,11 & 1,1800 \\
\hline
\end{tabular}

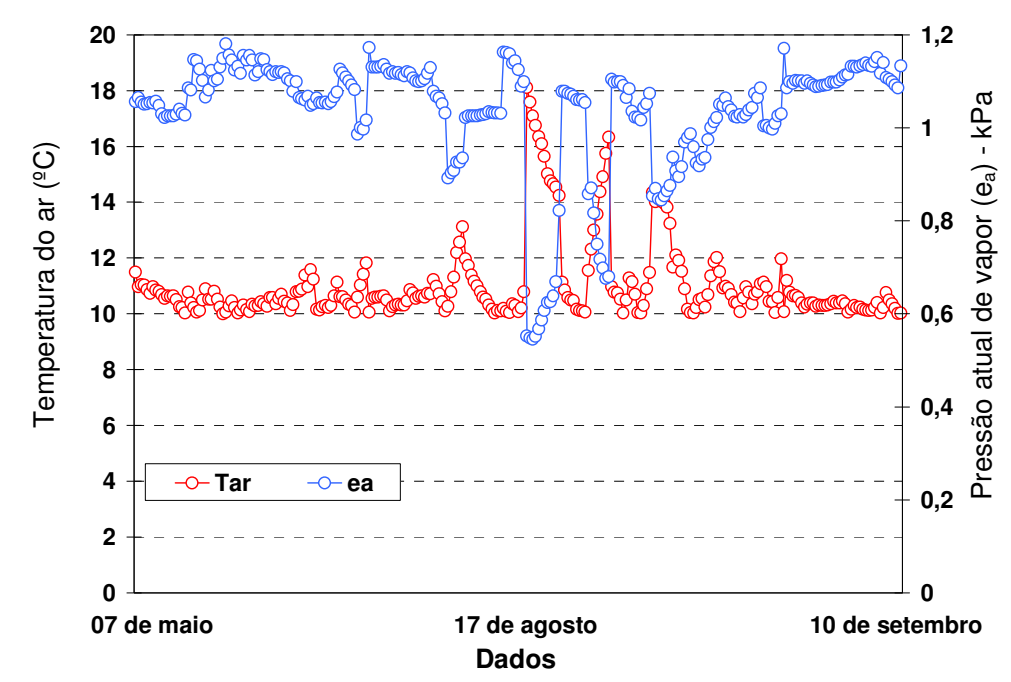

FIGURA 6. Análise dos 263 valores de $\mathrm{T}_{\mathrm{ar}}$ e de $\mathrm{e}_{\mathrm{a}}$, em que o Método de Newton-Raphson não convergiu para uma solução da equação psicrométrica.

Por outro lado, também se observa pela Figura 5 que alguns valores de $\mathrm{T}_{\mathrm{u}}$ obtidos pelo Método de Newton-Raphson foram superiores aos obtidos pelo da Secante. Na tentativa de explicar esse comportamento, elaborou-se um gráfico com os valores de $\mathrm{T}_{\mathrm{ar}}$ e de $\mathrm{e}_{\mathrm{a}}$ para os referidos valores; no total, foram 5.467 valores, nos quais o Método de Newton-Raphson superestimou os valores de $\mathrm{T}_{\mathrm{u}}$ em relação ao da Secante (Tabela 2). Pôde-se observar que o comportamento dos valores de $\mathrm{T}_{\mathrm{ar}}$ e de $\mathrm{e}_{\mathrm{a}}$ para que o Método de Newton-Raphson superestimasse os valores, deve-se principalmente ao fato de que os valores de $\mathrm{T}_{\mathrm{ar}}$ e $\mathrm{e}_{\mathrm{a}}$ foram maiores para o respectivo período (Figura 7).

Portanto, diante das simulações realizadas pela rotina computacional, pode-se evidenciar melhor desempenho do Método da Secante em relação ao de Newton-Raphson para a resolução numérica da equação psicrométrica. Isso se deve, principalmente, ao fato de que algumas situações 
combinadas entre a $\mathrm{T}_{\mathrm{ar}}$ e a $\mathrm{e}_{\mathrm{a}}$ fizessem com que o método de Newton-Raphson não convergisse para uma solução.

TABELA 2. Valores médios, mínimos e máximos de $\mathrm{T}_{\mathrm{ar}}$ e $\mathrm{e}_{\mathrm{a}}$ (5.467 dados), da série de 32.767 valores, de 2003, para Piracicaba - SP.

\begin{tabular}{ccc}
\hline & \multicolumn{2}{c}{ Elementos Climáticos } \\
\cline { 2 - 3 } & Temperatura do $\operatorname{Ar}\left(\mathrm{T}_{\mathrm{ar}}\right)$ & Pressão Atual de Vapor $\left(\mathrm{e}_{\mathrm{a}}\right)$ \\
$\mathrm{n}$ & ${ }^{\circ} \mathrm{C}$ & $\mathrm{kPa}$ \\
\hline Média & 19,41 & 0,6884 \\
Valor Mínimo & 5,93 & 3,390 \\
Valor Máximo & 35,23 & 3,398 \\
\hline
\end{tabular}

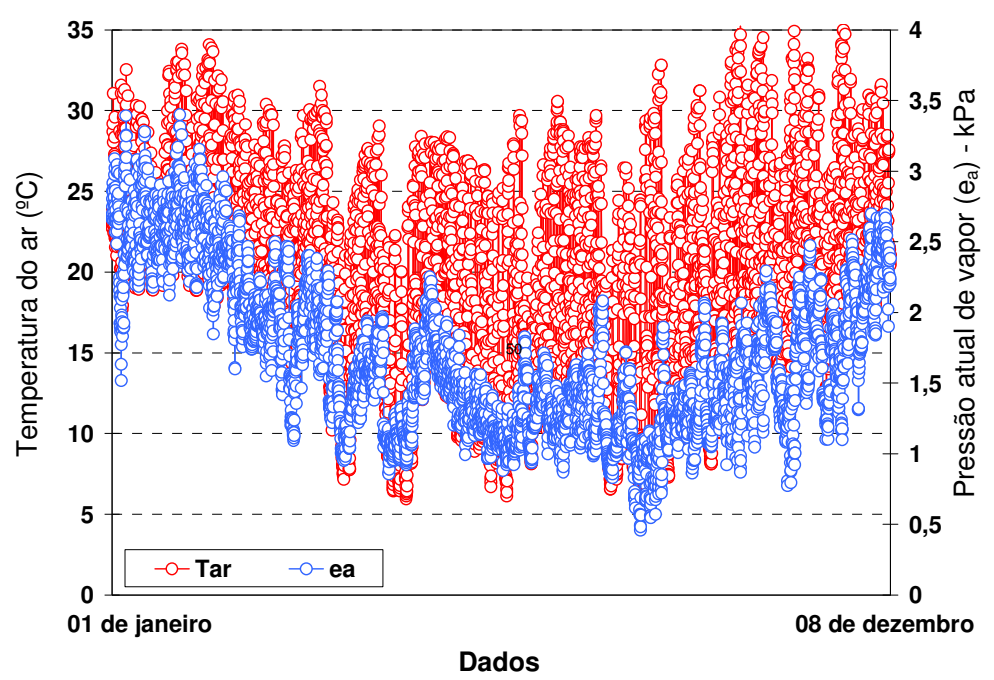

FIGURA 7. Análise dos 5.467 valores de $\mathrm{T}_{\mathrm{ar}}$ e de $\mathrm{e}_{\mathrm{a}}$, em que o Método de Newton-Raphson superestimou os valores de $\mathrm{T}_{\mathrm{u}}$ em relação ao da Secante.

\section{CONCLUSÕES}

Os métodos numéricos de Newton-Raphson e da Secante apresentaram bom desempenho na simulação dos resultados, porém o método da Secante foi melhor para ser aplicado à resolução numérica da equação psicrométrica.

Para valores baixos de $\mathrm{T}_{\mathrm{ar}}$ e de $\mathrm{e}_{\mathrm{a}}$, o método de Newton-Raphson encontrou dificuldades em solucionar a equação psicrométrica; para valores elevados de $\mathrm{T}_{\mathrm{ar}}$ e de $\mathrm{e}_{\mathrm{a}}$, o método de NewtonRaphson apresentou tendência de superestimar os valores de $T_{u}$ em relação ao da Secante.

\section{REFERÊNCIAS}

ASHRAE. ASHRAE Handbook-Fundamentals. Atlanta: American Society of Heating, Refrigerating and Air-Conditioning Engineers, 1997.

CASTELLVÍ, F.; PEREZ, P.J.; VILLAR, J.M.; ROSELL, J.I. Analysis of methods for estimating vapor pressure deficits and relative humidity. Agricultural and Forest Meteorology, Amsterdam, v.82, n.1, p.29-45, 1996.

COSTA, E. Medidas da umidade relativa do ar em um ambiente fechado. Revista Brasileira de Ensino de Física, São Paulo, v.25, n.3, p.346-8, 2003.

DIAS, J.B. Construção e validação de uma bancada para calibração de sensores para determinação da umidade relativa do ar. 2001. 153 f. Dissertação (Mestrado em Energia) Universidade Federal do Rio Grande do Sul, Porto Alegre, 2001. 
DÍEZ, P. A note on the convergence of the secant method for simple and multiple roots. Applied Mathematics Letters, St. Louis, v.16, n.8, p.1211-15, 2003.

JUPP, D.L.B. Calculating and converting between common water vapour measures in meteorological data and their use in support of earth observation. Camberra: CSIRO Earth Observation Centre Technical Report, 2003. 49 p.

MONTEITH, J.L.; UNSWORTH, M.H. Principles of environmental physics. $2^{\text {nd }}$ ed. London: Edward Arnold, 1990. 291 p.

MURRAY, F.W. On the computation of saturation vapour pressure. Journal of Applied Meteorology, Boston, v.6, N.1, 203-4, 1967.

PEREIRA, A.R.; ANGELOCCI, L.R.; SENTELHAS, P.C. Agrometeorologia: fundamentos e aplicações práticas. Guaíba: Livraria e Editora Agropecuária, 2001. 478 p.

SILVA, C.E. Comparação de painéis evaporativos de argila expandida e celulose para sistema de resfriamento adiabático do ar em galpões avícolas com pressão negativa em modo túnel. 2002. 77 f. Dissertação (Mestrado em Construções Rurais e Ambiência) - Universidade Federal de Viçosa, Viçosa - MG, 2002.

SINGH, A.K.; SINGH, H.; SINGH, S.P.; SAWHNEY, R.L. Numerical calculation of psychrometric properties on a calculator. Building and Environment, Oxford, v.37, n.4, p.415-19, 2002.

TETENS, O. Über einige meteorologische Begriffe. Zeitschrift Geophysic, Wurzburg, v.6, 297309, 1930. 